\title{
Implementation and alignment of a Raman spectroscopy array for powder and liquid samples
}

\section{Implementación y alineación de un equipo de espectroscopía Raman para muestras en polvo y líquidos}

\author{
A. Quiroz ${ }^{1 *}$, R. Sánchez ${ }^{1}$ \\ 1. Pontificia Universidad Católica del Perú, Departamento de Ciencias, Grupo de Óptica Aplicada, \\ Ap. Postal 1761, Lima 32, Perú \\ ${ }^{(*)}$ E-mail: aransselly.quiroz@pucp.pe \\ Received: 02/12/2016 \\ Accepted: $17 / 10 / 2017$ \\ DOI: $10.7149 /$ OPA.50.4.49046
}

\begin{abstract}
:
This paper presents the methodology for the implementation of a conventional Raman spectroscopy array for the qualitative analysis of powder and liquid samples. An alignment methodology has been established for a Raman array with $180^{\circ}$ geometry and sample collection on two axes. The array has been optimized to collect Raman signal in the 540 to $700 \mathrm{~nm}$ region ( $\Delta v: 160 \mathrm{~cm}^{-1}$ to $\left.4000 \mathrm{~cm}^{-1}\right)$ with integration times of up to 8 seconds without risk of Rayleigh band overlap.
\end{abstract}

Keywords: Raman spectroscopy, optical instrumentation, optical alignment.

\section{RESUMEN:}

En este trabajo se presenta la metodología de implementación de un equipo de espectroscopia Raman convencional para análisis cualitativo de muestras en polvo y líquidos. Se ha establecido un protocolo de alineación para un arreglo Raman con geometría $180^{\circ}$ y con colección a muestras en dos ejes. El arreglo se ha optimizado para colectar señal Raman en la región 540 a $700 \mathrm{~nm}\left(\Delta \mathrm{v}: 160 \mathrm{~cm}^{-1}\right.$ a 4000 $\mathrm{cm}^{-1}$ ) con tiempos de integración de hasta 8 segundos sin riesgo de superposición de la banda Rayleigh.

Palabras clave: Espectroscopía Raman, instrumentación óptica alineamiento óptico.

\section{REFERENCIAS Y ENLACES}

[1] BETTY CHUNG, "Control de los contaminantes químicos en el Perú," Scielo, 25, no. 4, (2008) [Online]. http://www.scielo.org.pe/scielo.php

[2] Ewen Smith and Geofry Dent, Modern Raman Spectroscopy - A Practical Approach, Primera ed., Antony Rowe and Chippenham Wiltshire, Eds. Chichester, England: John Wiley \& Sons, Ltd, (2005).

[3] John Ferraro, Kanzuo Nakamoto, and Chris w. Brown, Introductory Raman Spectroscopy, Segunda ed. California, Estados unidos de America: Academic Press, (2003).

[4] Douglas Skoog, James Holler, and Stanley Crouch, Principios de Análisis Instrumental, sexta ed., Sergio Cervantes Gonzales, Ed. México D.F., México: Cengage, (2008).

[5] Roberto Ysaac Sato Berrú, "Detección dce contaminantes en la agricultura mediante espectroscopía Raman," Centro de Investigaciones en Óptica, Guanajuato, Tesis de doctorado en ciencias (2003). 
[6] Richard Mc. Creery, Raman spectroscopy for chemical analysis, Primera ed. Canadá: Wiley Interscience, (2000).

[7] Paul Clemens, Xin Wei, Bobby L. Wilson, and Renard L. Thomas, "Anatase titanium dioxide coated single wall carbon nanotubes manufactured by sonochemical-hydrothermal technique," Open Journal of Composite materials, 3, pp. 21-32, (2013).

[8] Shuxia Guao, Zuliang Du, and Shuxi Dai, "Analysis of Raman modes in Mn-doped ZnO nanocrystals," Physica status solidi, 246, 10, pp. 2329-2332, (2009).

[9] Florian M. Zehentbauer, Euan J. Bain, and Johannes Kiefer, "Multiple parameter monitoring in a direct methanol fuel cell," Measurement Science and Technology, 23, 045602 (2012).

[10] Constanca Cacela, M.L. Duarte, and Rui Fausto, "Structural and vibrational characterisation of 3-amino-1propanol a concerted SCF-MO ab initio, Raman and infrared (matrix isolation and liquid phase) spectroscopy study," Spectrochimica Acta Part A, 56, pp. 1051-1064, (2000).

[11] ChemicalBooks. Chemical Books. [Online]. http://www.chemicalbook.com/ProductIndex EN.aspx

[12] Thorlabs. Thorlabs. [Online]. www.thorlabs.com

\section{INTRODUCCIÓN}

"En el Perú se produce una contaminación permanente del recurso hídrico tanto por productos químicos derivados de la minería y el narcotráfico, como por compuestos orgánicos derivados de las descargas domésticas, hospitalarias é industriales" [1]. La iniciativa de investigar en espectroscopía Raman se presenta con la finalidad de desarrollar equipos que permitan detectar contaminantes presentes en aguas crudas en tiempo real y que no requiera casi ninguna preparación de la muestra. Esta inquietud motivó la construcción de un arreglo (prototipo) de espectroscopía Raman para análisis cualitativo de muestras en polvo y muestras líquidas.

En la construcción del equipo, se han desarrollado dos procesos: 1) focalización del haz láser en la muestra de interés y 2) recolección de la radiación emitida por la muestra. [2], [3]. En ambos procesos, es importante realizar una correcta alineación de todos los elementos ópticos dispuestos en el equipo, así, se reducen las pérdidas de radiación emitida por la muestra y se optimiza la recolección de fotones Raman que llegan a ser registrados por el espectrómetro.

El contenido de este documento es como sigue: en la sección 2, se aborda el fundamento teórico de la espectroscopía Raman, en la sección 3, se detalla el proceso de montaje y alineamiento de los elementos ópticos dispuestos en el arreglo, en la sección 4, se presentan los resultados de la calibración y validación del prototipo Raman, finalmente, la sección 5 corresponde a las conclusiones de este trabajo.

\section{FUNDAMENTO TEÓRICO}

Como resultado de exponer una sustancia a la radiación electromagnética, sus moléculas pueden absorber, emitir o dispersar energía; cada uno de estos procesos lleva consigo transferencia de energía que resulta de la interacción de la nube de electrones de dicha molécula con la fuente de radiación. Así, mientras en los procesos de absorción y emisión están asociados saltos cuánticos entre niveles electrónicos, vibracionales ó rotacionales cuyos tiempos de vida son de: $10^{-8} \mathrm{~s}, 10^{-15} \mathrm{~s}$ y $10^{-11} \mathrm{~s}$ respectivamente [4]; en el proceso de dispersión no existen tales saltos, lo que ocurre es que la molécula se polariza (polarización inducida) y alcanza temporalmente $\left(10^{-14} \mathrm{~s}\right)$ un nivel de energía superior denominado estado virtual, cuya energía es menor al primer estado electrónico [4], [2]. La figura 1 muestra un esquema de la polarización inducida en una molécula por acción de un campo eléctrico externo. 


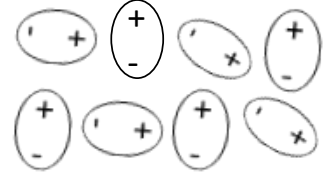

a) Moléculas neutras

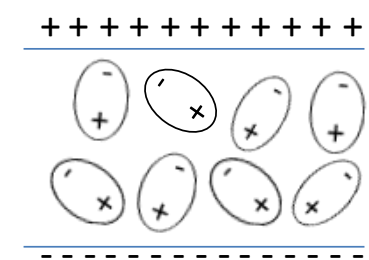

b) Moléculas expuestas a un $\mathbf{E}_{\text {láser }}$

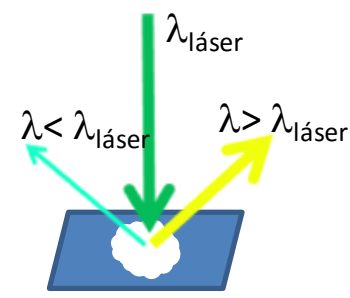

c)

Fig. 1. Esquema de polarización inducida en una molécula: a) Las moléculas se encuentran en estado neutro, b) Moléculas expuestas a un campo eléctrico, c) Emisión de radiación de la molécula $\left(\lambda<\lambda_{\text {láser, }}, \lambda>\lambda_{\text {láser }}\right)$ debido a la acción de un campo eléctrico externo $\left(\lambda_{\text {láser }}\right)$.

La polarización inducida $P$ está definida por:

$$
P_{\text {inducido }}=\alpha E_{\text {láser }}
$$

donde: $\alpha$ es la constante de proporcionalidad llamada polarizabilidad y $E_{\text {láser }}$ es el campo eléctrico de la radiación indicente. Tanto $\alpha$ como $E_{\text {láser }}$ están definidas por:

$$
\begin{gathered}
\alpha=\alpha_{0}+(\partial \alpha / \partial q)_{0} q \\
E_{\text {láser }}=E_{0} \cos \left(2 \pi v_{0} t\right)
\end{gathered}
$$

En la ecuación (2), $q$ es el desplazamiento nuclear que se produce como resultado de la vibración a lo largo del enlace que conecta los núcleos y $\alpha_{0}$ es la polarizabilidad a la posición de equilibrio.

En la ecuación (3), $v_{0}$ es la frecuencia de la radiación incidente y $E_{0}$ es la amplitud del campo eléctrico de la radiación incidente.

Reemplazando (2) y (3) en (1) se obtiene una expresión del momento dipolar inducido $P$ y está dado por:

$$
P=\alpha_{0} E_{0} \cos \left(2 \pi v_{0} t\right)+\frac{1}{2}\left(\frac{\partial \alpha}{\partial q}\right)_{0} q_{0} E_{0}\left[\begin{array}{c}
\cos \left(2 \pi\left(v_{0}+v_{m}\right) t\right)+ \\
\cos \left(2 \pi\left(v_{0}-v_{m}\right) t\right)
\end{array}\right]
$$

En la ecuación (4), el primer término representa un dipolo oscilante que radia luz de frecuencia $v_{0}$ (dispersión Rayleigh), mientras el segundo término corresponde a la dispersión Raman de frecuencia $v_{0}+v_{m}$ (anti-Stokes) y $v_{0}-v_{m}$ (Stokes). Si $(\partial \alpha / \partial q)_{0}$ es cero, la vibración no es activa en Raman [3].

Para que los términos de la derivada $(\partial \alpha / \partial q)_{0}$ no sean cero, al menos uno de los componentes de la polarizabilidad: $\alpha_{x x}, \alpha_{x y}, \ldots$ debe de cambiar durante la vibración descrita por " $q$ " [5].

Pero ¿qué cambios ocurren en la molécula?, básicamente se refiere a cambios en su energía vibracional. Desde el punto de vista de la mecánica clásica la energía está dada por:

$$
E=E_{\text {cinética }}+E_{\text {potencial }}=\left(\frac{1}{2} \mu\left(\frac{d q}{d t}\right)^{2}\right)+\left(\frac{1}{2} K q^{2}\right)=\text { constante }
$$

De la ecuación (5), se observa que $E$ tiene una dependencia cuadrática con $q$, lo cual nos indica que la energía de un estado vibracional puede variar continuamente. Sin embargo, a escala atómica, esta relación deja de ser válida y se cumple los principios de la mecánica cuántica. 
Según la mecánica cuántica: "Si una molécula interactúa con un campo eléctrico, una transferencia de energía del campo a la molécula puede ocurrir únicamente cuando la condición de Bohr es satisfecha, es decir, se cumple que $\Delta E=E_{2}-E_{1}=h v=h(c / \lambda)=h c \bar{v}$.

En los fenómenos de dispersión Raman ocurre un cambio de energía en la molécula cuando interacciona con un fotón de la fuente. El aumento de energía es igual a la energía del fotón $h v$ de la fuente de excitación (láser), por lo tanto, dependiendo de la frecuencia de la radiación de la fuente, la energía de la molécula puede tomar cualquiera de los infinitos valores entre el estado fundamental y el primer estado electrónico excitado (algunos autores lo llaman "estado virtual") [4]." La desexitación de la molécula se puede dar a cualquiera de los niveles vibracionales del estado fundamental y va acompañada de la emisión de un fotón cuya energía dependerá del nivel vibracional al que decae la molécula. La figura 2 muestra un esquema de este proceso.

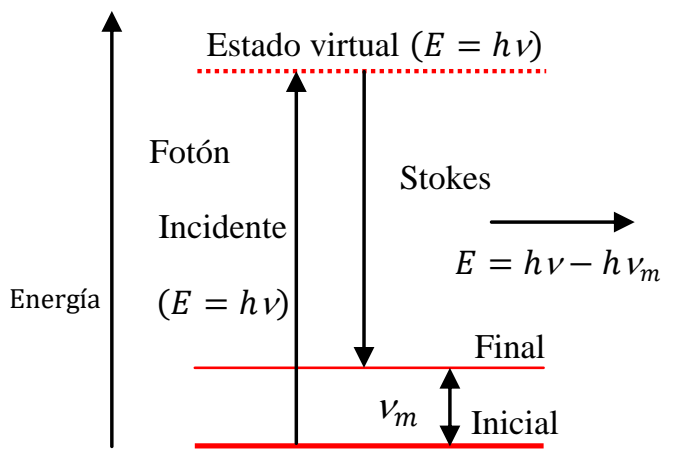

a)

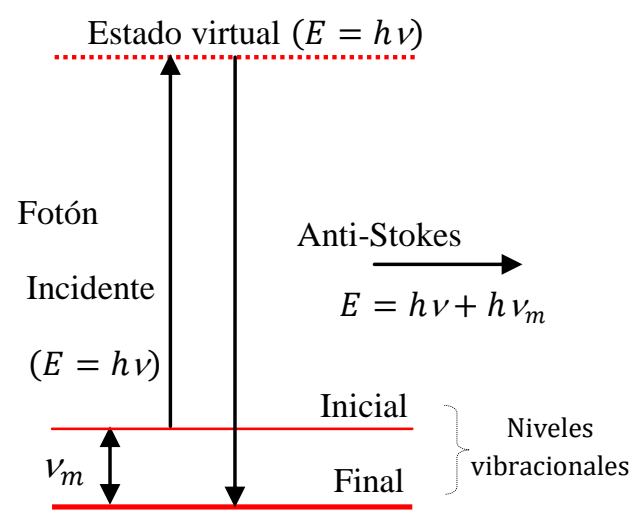

b)

Fig. 2. Esquema de los niveles de energía para la dispersión Raman: a) Dispersión Stokes, b) Dispersión Anti-Stokes. (Adaptado de [5]).

El conjunto de todos los fotones dispersados por la molécula son representados en una gráfica de intensidad versus longitud de onda $(\lambda=c / v)$, en la que se distingue la dispersión Rayleigh y la dispersión Raman Stokes y anti-Stokes. La figura 3 muestra la firma espectral de la molécula del azufre en polvo.
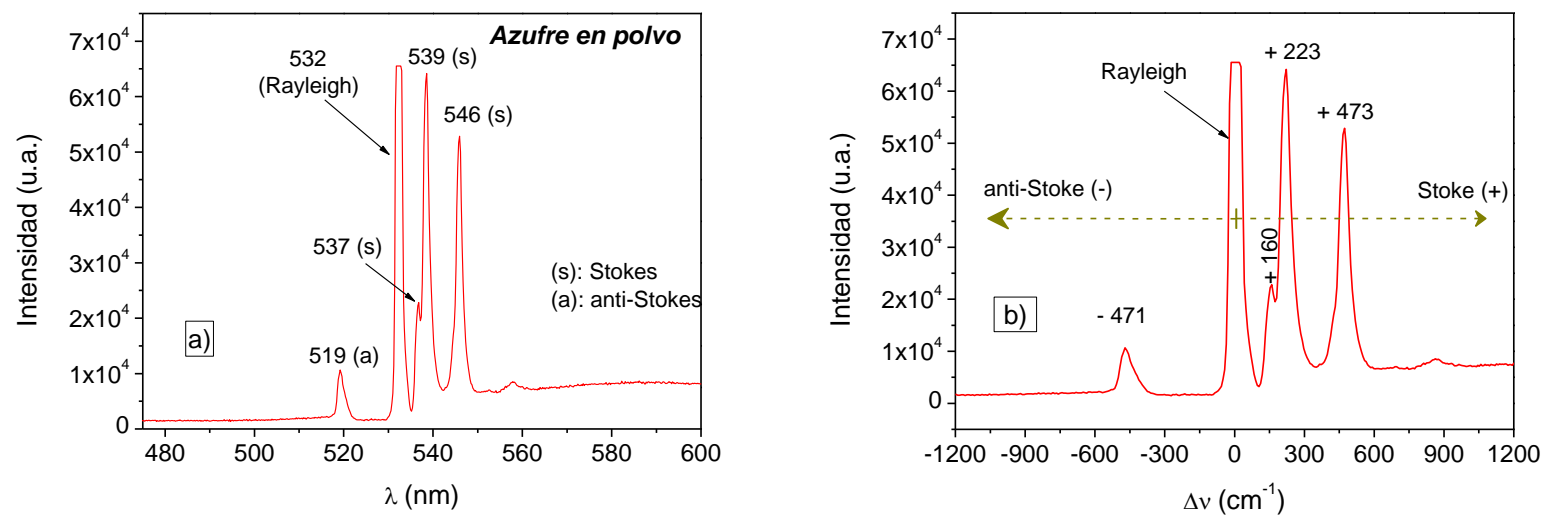

Fig. 3. Registro espectral de dispersión del azufre en polvo: a) En función de la longitud de onda, b) En función del desplazamiento Raman.

En [6], la intensidad de la dispersión Raman anti-Stokes depende de la población del primer estado vibracional excitado y está relacionada con la temperatura de acuerdo a la distribución de Boltzman: $\frac{I_{R}\left(v_{0}+v_{j}\right)}{I_{R}\left(v_{0}-v_{j}\right)}=$ $\frac{\left(v_{o}+v_{j}\right)^{4}}{\left(v_{0}-v_{j}\right)^{4}} e^{\left(\frac{-h v_{j}}{k T}\right)}$,además, la intensidad Raman Stokes y anti-Stokes $\left(I_{R}\right)$ tienen una dependencia cuadrática con 
la polarizabilidad de la molécula $\left(\alpha_{j}\right)$ y el desplazamiento $\left(q_{j}\right)$ según la siguiente expresión: $I_{R}=$ $\mu\left(v_{0} \pm v_{j}\right)^{4} \alpha_{j}^{2} q_{j}^{2}$. Estas variables son propias de cada molécula.

Hay otros factores que determinan la cantidad de fotones emitidos con dispersión Raman, los cuales están relacionados con la intensidad específica en la interacción muestra-láser (factor L), las características del detector (factor C) y la medida de tiempo para el valor particular del desplazamiento Raman que está siendo observado (factor t) [6].

$$
L=P_{D} \beta D k
$$

$P_{D}$ es la densidad de potencia del láser, $\beta$ es el rango de ángulo solido observado en una de las direcciones dispersadas, $D$ es la densidad de la dispersión Raman (en moléculas $/ \mathrm{cm}^{3}$ ), $k$ es la profundidad de campo del láser en la muestra.

$$
C=A_{D} \Omega_{D} T Q
$$

siendo: $A_{D}$ el área de la muestra monitoreada (usualmente está limitada a la apertura del espectrómetro), $\Omega_{D}$ es el ángulo sólido en estereorradianes colectado por el espectrómetro, T es la fracción de luz monitoreada que alcanza al detector y que se monitorea en la región entre $A_{D}$ y $\Omega_{D}$, Q es la eficiencia cuántica del detector y se expresa en ( $e^{-} /$foton $)$.

$$
t=t_{s}
$$

$t_{s}$ es el tiempo en que el valor del desplazamiento Raman de un modo de vibración particular está siendo observado.

La señal Raman total de la muestra de interés $(S)$, se determina a partir del producto de las ecuaciones (6), (7) y (8):

$$
S=P_{D} \beta D k \cdot A_{D} \Omega_{D} T Q \cdot t_{S}
$$

\section{IMPLEMENTACIÓN Y ALINEAMIENTO DEL ARREGLO}

\section{Configuración $\mathbf{1 8 0}^{\circ}$}

La literatura reporta diferentes geometrías para equipo Raman, una de ellas establece que el eje del láser y el eje de colección sean coincidentes para facilitar su reproducibilidad, es la denominada geometría $180^{\circ}$ [6] y es la que se propone en este proyecto.

La figura 4 muestra un esquema de esta geometría, en la cual el haz láser es coaxial con el eje de colección "y" y eje de colección " $z$ ".

Esta geometría tiene un divisor de haz 40:60 y le ofrece al equipo Raman la capacidad de analizar muestras en ambas salidas del divisor.

Ya que solo 1 de cada 106 fotones son emitidos con radiación Raman, es preciso optimizar la colección de la radiación emitida optimizando la alineación del haz láser para reducir las pérdidas de radiación por desviaciones del haz durante todo su recorrido óptico.

La alineación del equipo se inicia poniendo a nivel cada una de las monturas que soportan a los elementos ópticos, para ello, se tomó como referencia el tablero óptico que soporta el equipo. 


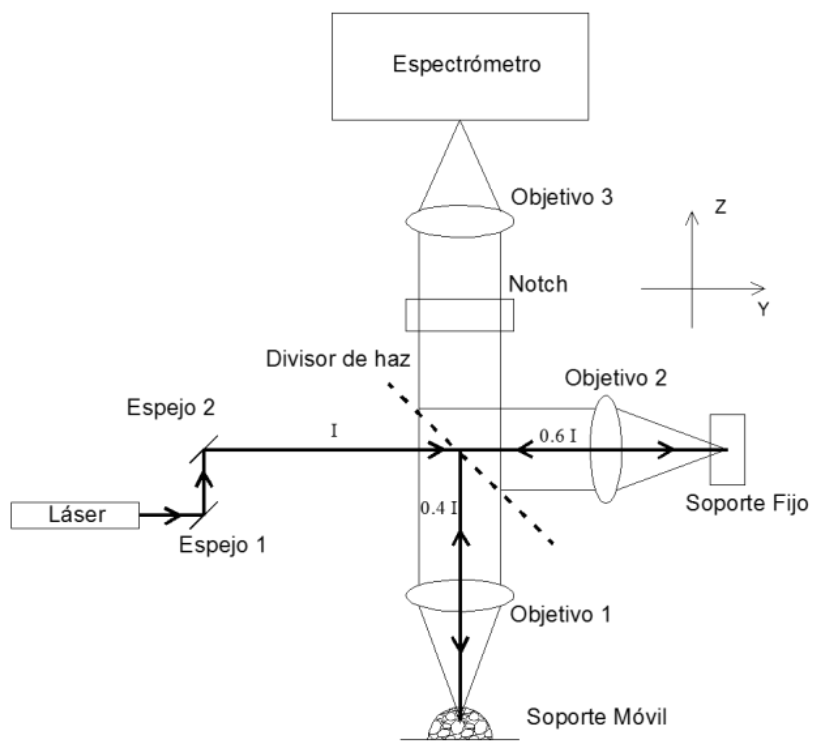

Fig. 4. Esquema de la geometría del equipo Raman en configuración $180^{\circ}$.

\section{Óptica de dirección y alineación del haz láser en el plano horizontal.}

La figura 5 muestra la disposición de los elementos ópticos empleados en la alineación del haz láser en el plano horizontal. De izquierda a derecha, se tiene: un láser doblado en frecuencia de $532 \mathrm{~nm}$, dos espejos dicroicos (reflexión a 532nm) y un divisor de haz 40:60 con cubo. Cada elemento óptico se encuentra soportado en monturas mecánicas y cinemáticas que permiten movimientos de traslación y rotación del haz hasta en 3 grados de libertad.

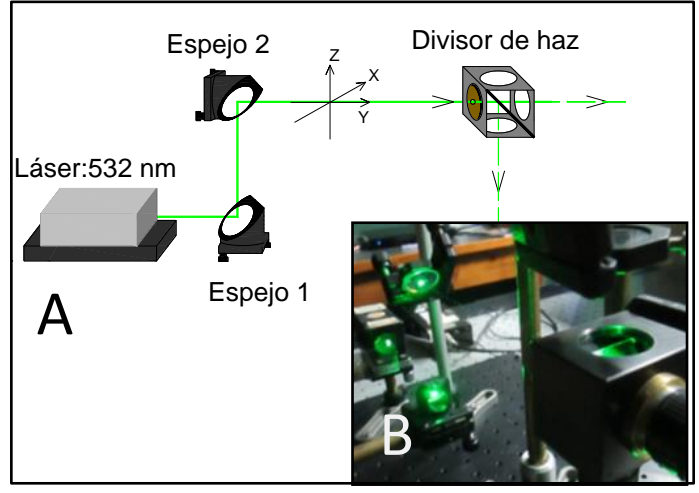

Fig. 5. (A) Disposición de elementos ópticos para alineación del láser en el plano horizontal (eje y), (B) Imagen de los elementos ópticos mostrados en A.

La disposición de los espejos es de reflexión a $90^{\circ}$. Estos espejos son dicroicos o también denominados separadores armónicos, permiten separar la radiación láser (láser doblado de $\mathrm{Nd}$ : $\mathrm{YVO}_{4}$ ) en sus componentes armónicas: $1064 \mathrm{~nm}$ y $532 \mathrm{~nm}$.

El siguiente paso fue direccionar el haz láser paralelamente en el eje " $y$ ". Las desviaciones del haz que se generan alrededor de los ejes " $\mathrm{x}$ ", " $\mathrm{z}$ " se corrigen ajustando la orientación del par de espejos dicroicos E1 y E2, los cuales se encuentran soportados en monturas cinemáticas que le permiten desplazar el haz láser sobre el PH1 en dos grados de libertad: traslación en el eje x, traslación en el eje z. 
Los soportes de los espejos dicroicos cuentan con perillas giratorias para orientar el haz. Las perillas P2 y P3 permiten orientar el haz sobre el eje " $\mathrm{x}$ " y las perillas P1 y P4 permiten orientar el haz sobre el eje " $z$ ". Véase la figura 6 .

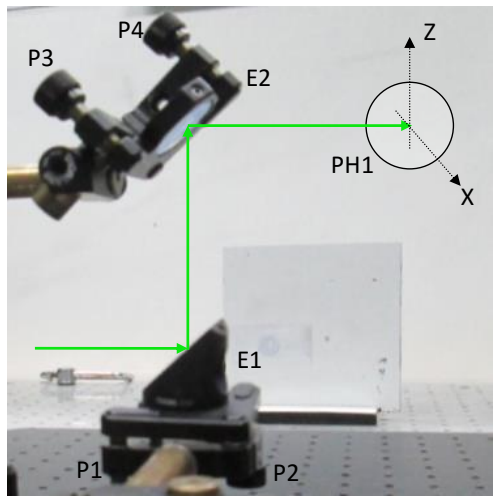

Fig. 6. Disposición de los espejos dicroicos: E1 y E2 para alinear el haz láser en dos grados de libertad: “z”, "x”. El haz debe pasar a través del pinhole 1 .

La posición de los espejos queda definida cuando el haz láser pasa a través de los pinhole 1 , pinhole 2 y pinhole 4 sin sufrir desviaciones en todo su recorrido. Entre el pinhole 2 y 3 se encuentra el separador de haz que divide el haz en dos partes: una parte en la dirección horizontal pasa por el pinhole 4 y la otra parte, en dirección perpendicular, pasará por el pinhole 5, esto es, en la dirección vertical del equipo Raman. La figura 7 muestra la disposición de los pinhole (PH) en el equipo

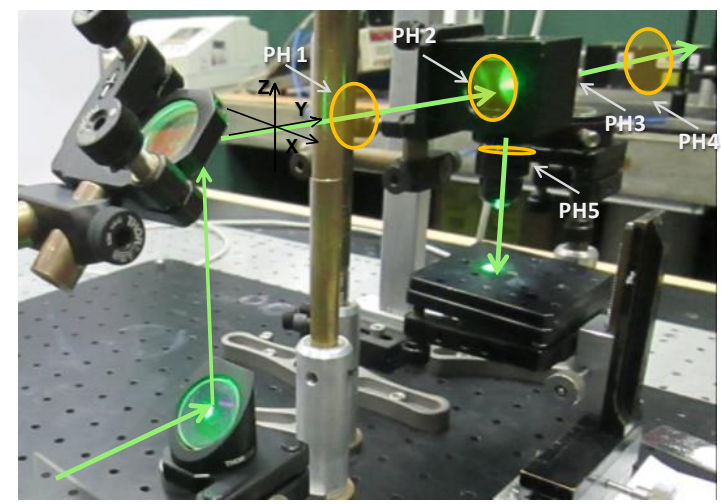

Fig. 7. Alineamiento del láser en el eje horizontal (eje y) y en el eje vertical (eje z) por acoplamiento de los pinhole 1 al 5 (diám etro de los pinhole: $2 \mathrm{~mm}$ ).

\section{Óptica de dirección y alineación del haz láser en el plano vertical.}

El equipo dispone de dos soportes portamuestras a ambas salidas del haz láser, una para muestras sólidas ó en portaobjetos y otra para muestras líquidas, la misma que podría ser removida en caso que se requiera analizar objetos de gran tamaño, superior a los $10 \mathrm{~cm}$ de altura. La figura 8 muestra la disposición de las plataformas portamuestras.

El primer paso en la alineación del eje vertical (eje z), consistió en nivelar los dos portamuestras tomando como referencia el cubo que soporta el separador de haz. Luego, se instalaron dos pinhole: PH6 y PH7 en la parta superior del cubo y se dispuso un espejo auxiliar plano sobre cada portamuestras para verificar por reflexión 
el retorno del haz láser hacia el pinhole 5 y que continúe su recorrido pasando por los pinhole 6 y 7 . La figura 8 muestra esta disposición.

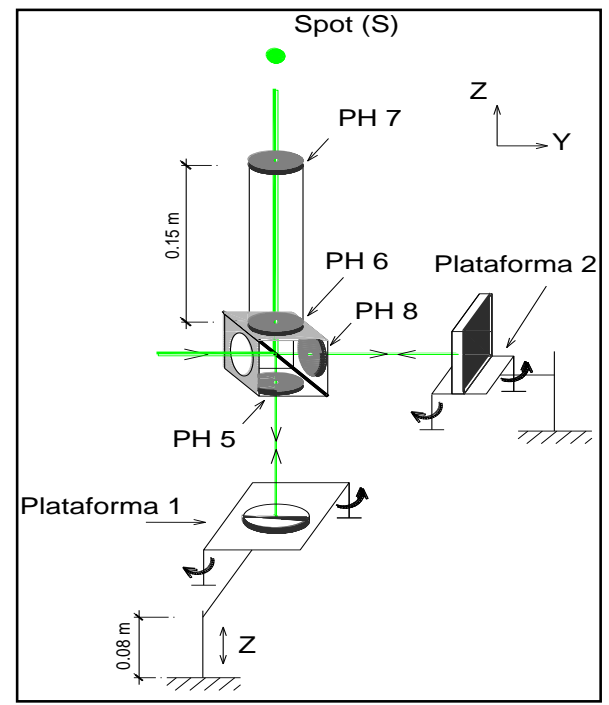

Fig. 8. Esquema de disposición de plataformas portamuestras para alineación del láser por reflexión en el eje vertical (eje z). Los pinhole están denotados como PH.

\section{Óptica de focalización a muestras y recolección Raman}

En esta parte del alineamiento óptico, interesa determinar la longitud focal " $f$ " de las lentes para optimizar la detección de la señal Raman que está dada por la expresión (9), en la que $S=P_{0} \beta D\left(K \frac{A_{D}}{A_{L}} \Omega_{D}\right) T Q t_{s}$.

El producto de todas las variables de esta ecuación que son afectadas por la longitud focal "f" es: $K \frac{A_{D}}{A_{L}} \Omega_{D}$. Para una muestra compacta, este producto varía con $\frac{1}{f}$ y para una muestra delgada el producto varía con $\frac{1}{f^{2}}$ [6].

Según este análisis se optó por instalar lentes objetivos de corta longitud focal y se calculó el área mínima se sección transversal del láser sobre la muestra. El diámetro mínimo del spot del láser sobre la muestra tiene una dependencia lineal con la longitud focal de la lente y la divergencia del haz de acuerdo a la siguiente expresión: $\emptyset_{\text {min }}=f \theta_{d}$.Siendo: f la longitud focal de la lente de enfoque y $\theta_{\mathrm{d}}$ el ángulo de divergencia del láser en radianes [6].

La tabla 1 muestra los resultados obtenidos para los dos objetivos de focalización L1 y L2 que se instalaron a la salida del separador de haz (véase figura 9)

Tabla 1. Diámetro y área del láser sobre la muestra

\begin{tabular}{|c|c|c|c|c|}
\hline Lentes & $\begin{array}{c}\mathrm{f} \\
(\mathrm{mm})\end{array}$ & $\begin{array}{c}\theta_{d} \\
(\mathrm{mrad})\end{array}$ & $\begin{array}{c}\emptyset_{\min } \\
(\mu \mathrm{m})\end{array}$ & $\begin{array}{c}\left(A_{L}\right)_{\min } \\
\left(\mu \mathrm{m}^{2}\right)\end{array}$ \\
\hline 1 & 8 & 1.5 & 12 & 113 \\
\hline $\mathrm{L}_{2}$ & 9 & 1.5 & 13.5 & 143 \\
\hline
\end{tabular}




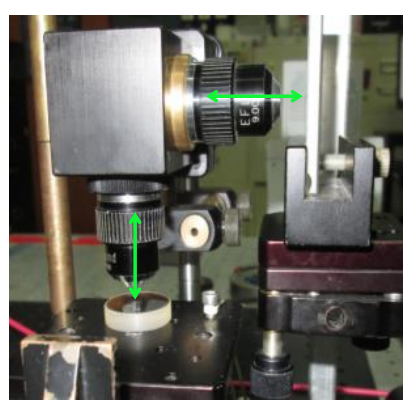

Fig. 9. Disposición de los objetivos para focalización del láser en la muestra y recolección Raman.

La recolección Raman al espectrómetro se desarrolló por acoplamiento de una lente de cámara fotográfica cuyos parámetros fueron calculados experimentalmente. La lente seleccionada tiene una distancia de trabajo de $9 \mathrm{~mm}$ y apertura numérica de $0.29\left(34^{\circ}\right)$ mayor a la del espectrómetro de $0.22\left(24^{\circ}\right)$, lo que genera un acoplamiento del tipo overfilled. La figura 10 presenta un esquema del sistema óptico de recolección, la región de color rojo representa las pérdidas de radiación por acoplamiento overfilled. La figura 11 muestra el acoplamiento lente-espectrómetro.

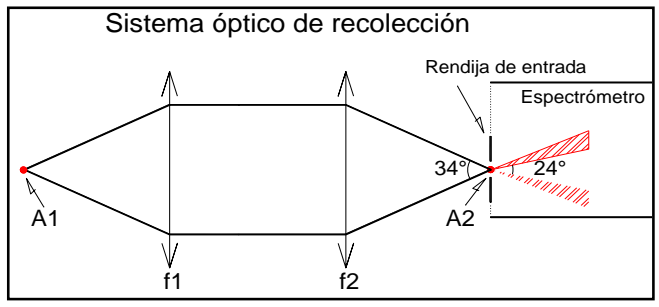

Fig. 10. Esquema del sistema óptico de recolección del arreglo Raman. f1 corresponde a la lente de focalización en la muestra y f2 corresponde a la lente de acoplamiento al espectrómetro.

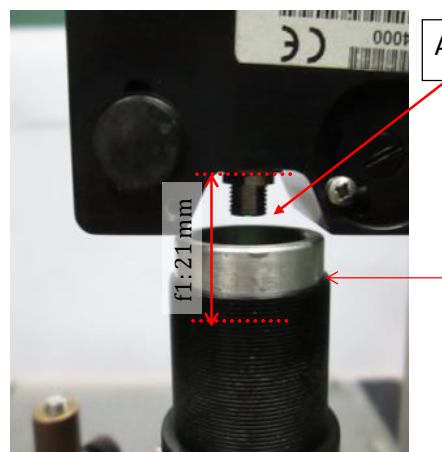

a)

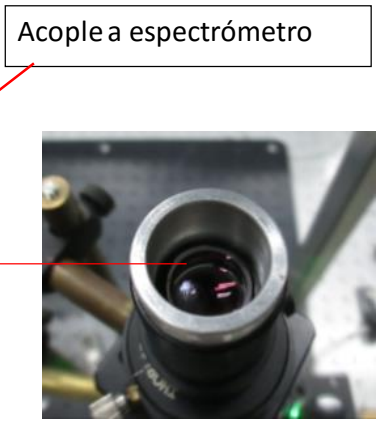

b)

Fig. 11. a) Se muestra el objetivo acoplado al espectrómetro, b) Vista desde arriba de una sección del objetivo.

Se filtró la señal Rayleigh que debido a su elevada intensidad: $10^{6}$ veces más que la señal Raman, puede provocar dos efectos: saturación del espectrómetro y, superposición del espectro Rayleigh sobre el espectro Raman. Para eliminar esta radiación, se instaló un filtro notch de $533 \mathrm{~nm} \pm 2 \mathrm{~nm}$ entre el separador de haz y el objetivo de acoplamiento a espectrómetro. Las figuras 12 a) y 12 b), muestran la posición del filtro en el arreglo Raman y las curvas características del filtro respectivamente. En la figura 12 b), las curvas de transmisión y densidad óptica del filtro Notch se tomaron del fabricante (Ver referencia [12]). 


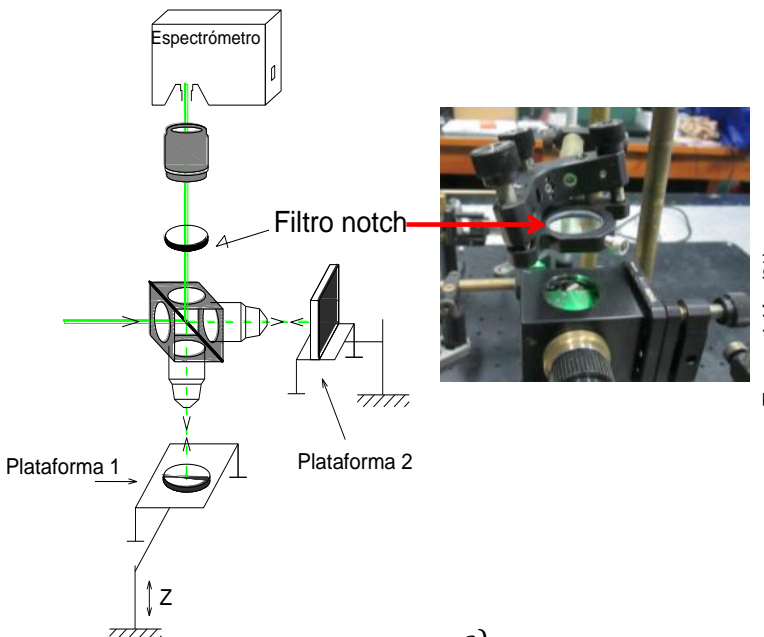

a)
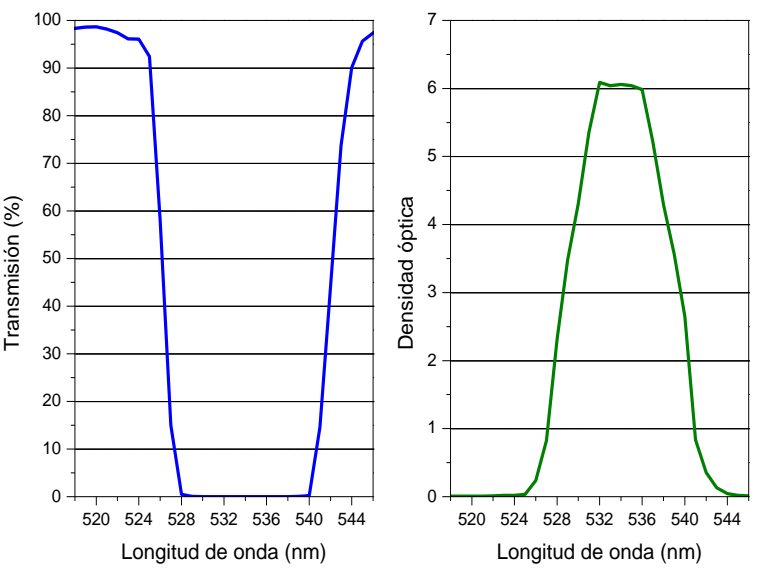

b)

Fig. 12. a) Disposición de filtro notch en el arreglo Raman, b) Curvas de transmisión y densidad óptica del filtro notch: NF533-14

\section{Alineamiento del espectrómetro a la óptica}

En esta etapa se optimiza la alineación del espectrómetro por observación de la firma espectral de sustancias en polvo y sustancias líquidas depositadas en las plataformas 1 y 2 respectivamente. El parámetro de observación es la intensidad espectral debido a la dispersión Rayleigh y Raman. Con ayuda de las perillas de ajuste fino de las monturas que soportan el espectrómetro, se optimiza su posición hasta obtener una firma espectral con intensidades pico máximas.

El primer paso en esta etapa consiste en detectar la radiación emitida por una muestra altamente fluorescente posicionada en la plataforma 2 del equipo, es el caso de la rodamina 6G. El segundo paso en esta etapa consiste en detectar la dispersión Rayleigh, por ser esta señal de mayor intensidad respecto de la señal Raman, pero de menor intensidad respecto de la señal de fluorescencia (véase figura 13 a), 13 b), 13 c)).

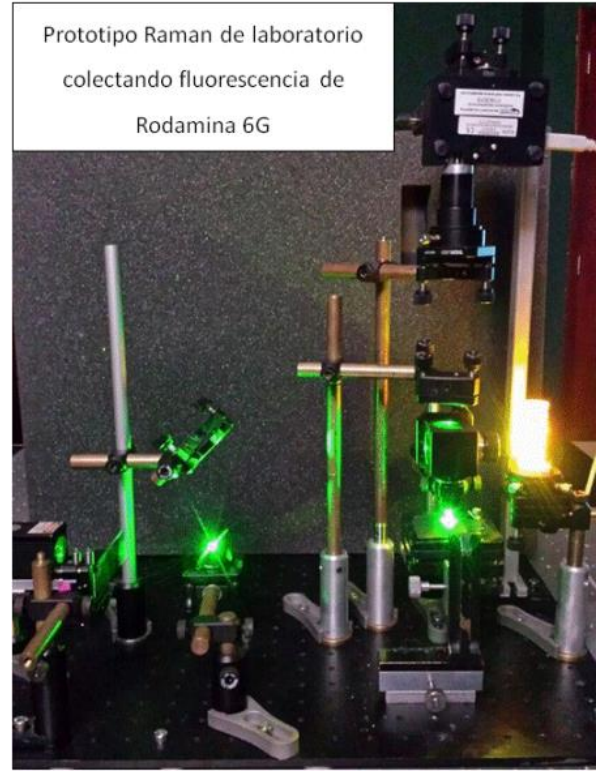

a)

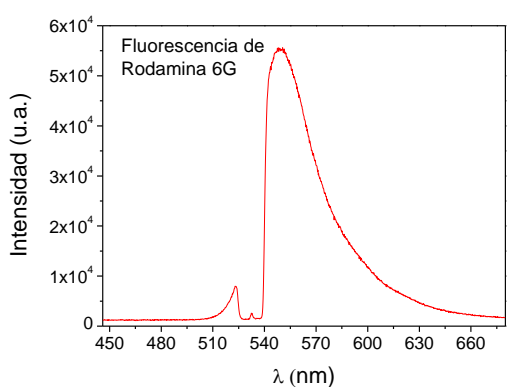

b)

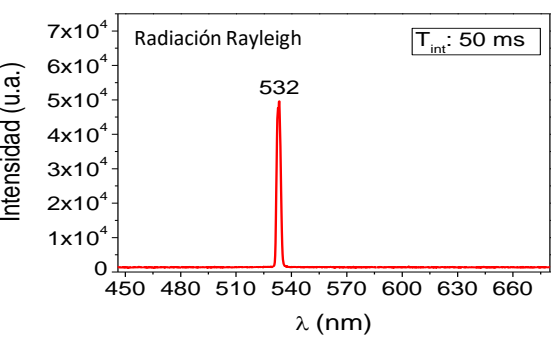

c)

Fig. 13. a) Arreglo Raman con una muestra de Rodamina 6G (cubeta en plataforma 2), b) Espectro de fluorescencia de Rodamina 6G, c) Espectro de radiación Rayleigh. 
El tercer paso consiste en detectar la dispersión Raman de muestras de laboratorio (muestras puras). Se replicó el procedimiento abordado con la dispersión Rayleigh en muestras de óxido de titanio (Clemens, Wei, Wilson, \& Thomas, 2013), óxido de zinc (Guao, Du, \& Dai, 2009) y agua desionizada (Zehentbauer, Bain, \& Kiefer, 2012), cuyos espectros se muestran en las figuras $14 \mathrm{a}), 14 \mathrm{~b}), 14 \mathrm{c}$ ).

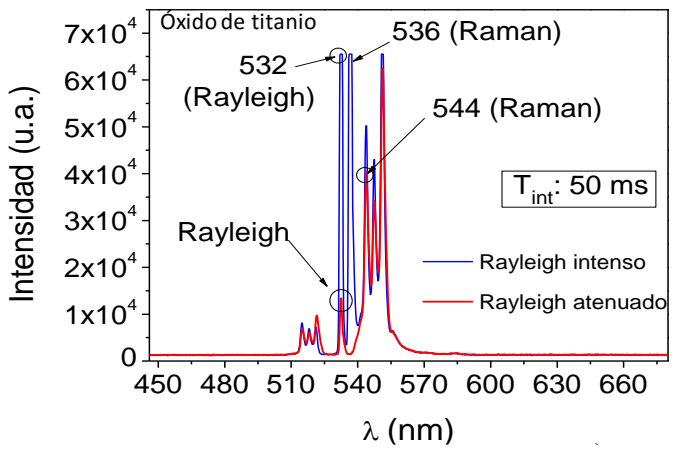

a)

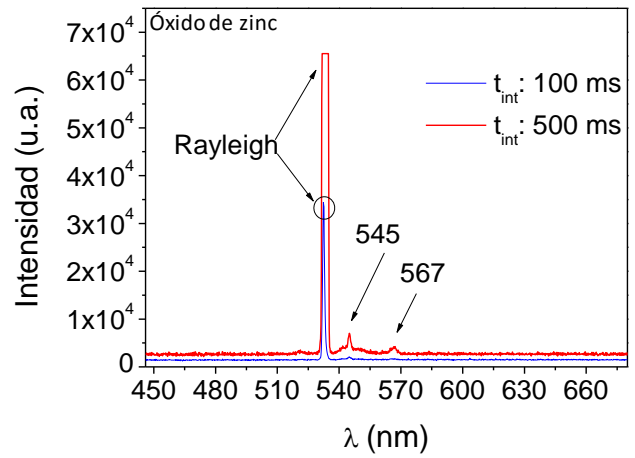

b)

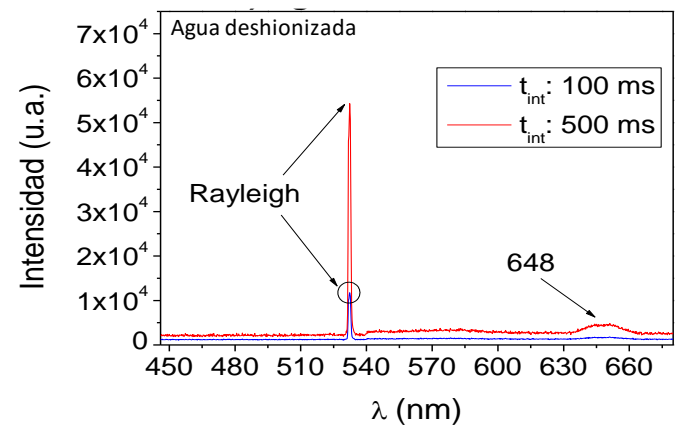

c)

Fig. 14. Evolución en el alineamiento del espectrómetro a la óptica del arreglo. Los espectros se colectaron con tiempos de integración de: a) 50 ms para el óxido de titanio, b) 100 y 500 ms para el óxido de zinc, c) 100 y 500 ms para el agua deshionizada.

\section{CALIBRACIÓN Y VALIDACIÓN}

La calibración del equipo se ha desarrollado con la técnica de desplazamiento en frecuencia Raman y se ha empleado una muestra en polvo de TiO (modos: $399 \mathrm{~cm}^{-1}, 524 \mathrm{~cm}^{-1}$ y $642.0 \mathrm{~cm}^{-1}$ ). En la mayoría de los casos, las diferencias entre el valor pico experimental y el valor pico reportado en la literatura $\left(398 \mathrm{~cm}^{-1}, 516 \mathrm{~cm}^{-1}\right.$ y 639 $\mathrm{cm}^{-1}$ ) fue menor a $4 \mathrm{~cm}^{-1}$ [6]. La figura 15 muestra los modos vibracionales del óxido de titanio [7]

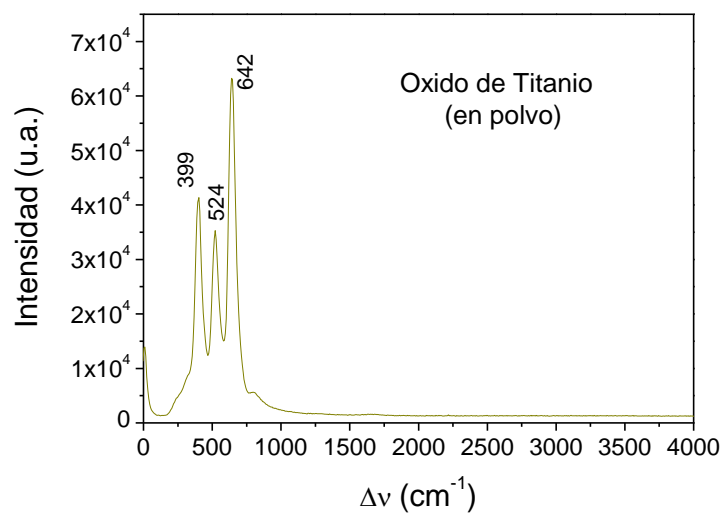

Fig. 15. Espectros Raman del óxido de Titanio en polvo. 
La validación del arreglo de espectroscopía Raman se desarrolló por comparación de los desplazamientos Raman de muestras de alcoholes, con la información existente en la literatura ( [10], [11], [9]). Las figuras 16 a), 16 b), 16 c) y 16 d) muestran los espectros capturados con el equipo Raman, se verifica que todas las muestras de alcohol contienen los grupos funcionales: C-C y C-O en la región 600-1500 cm-1, denominada región de la huella dactilar, el grupo C-H en la región entre 2800-3200 cm-1 y el grupo 0-H en la región 3200$3400 \mathrm{~cm}-1$.

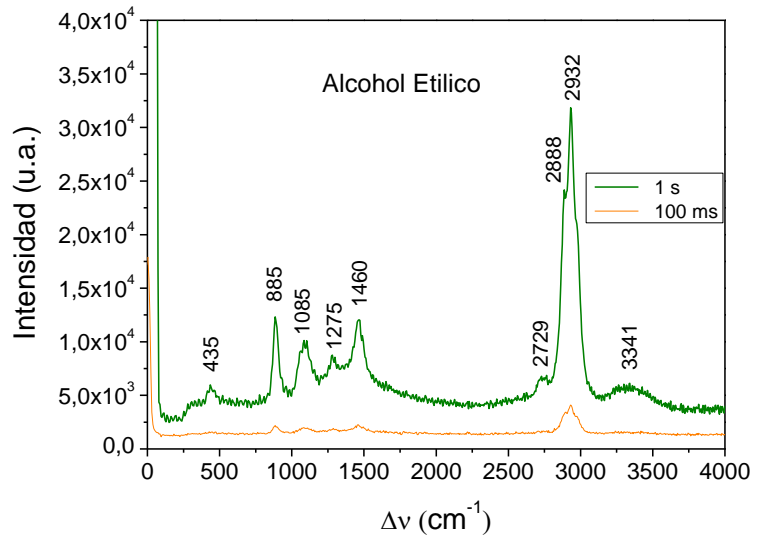

a)

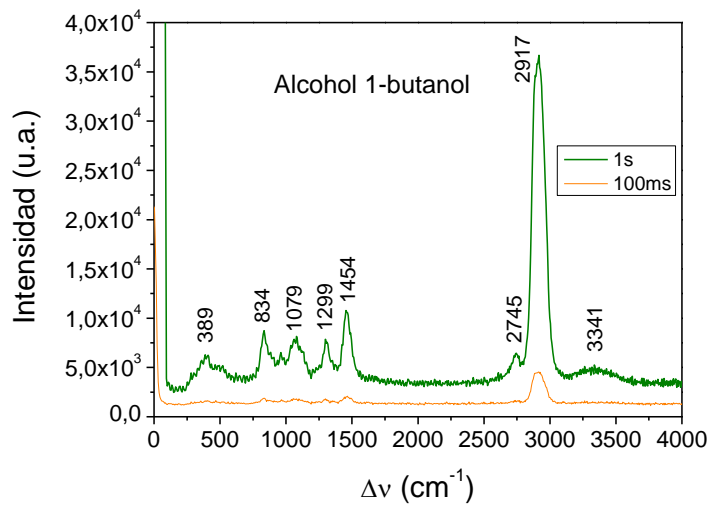

c)

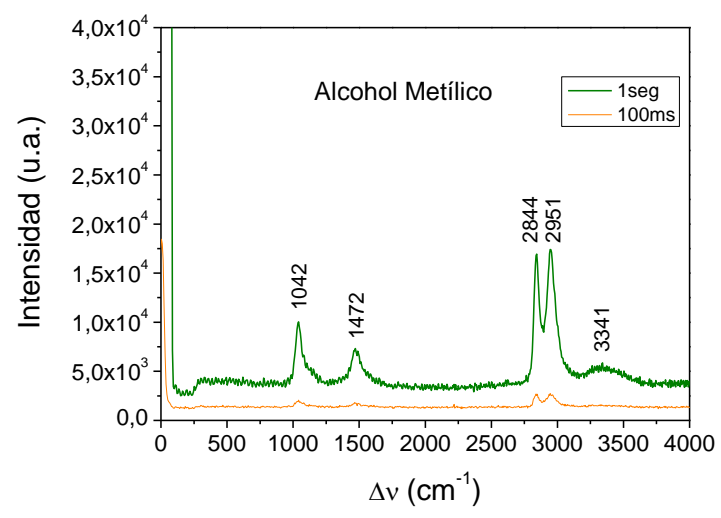

b)

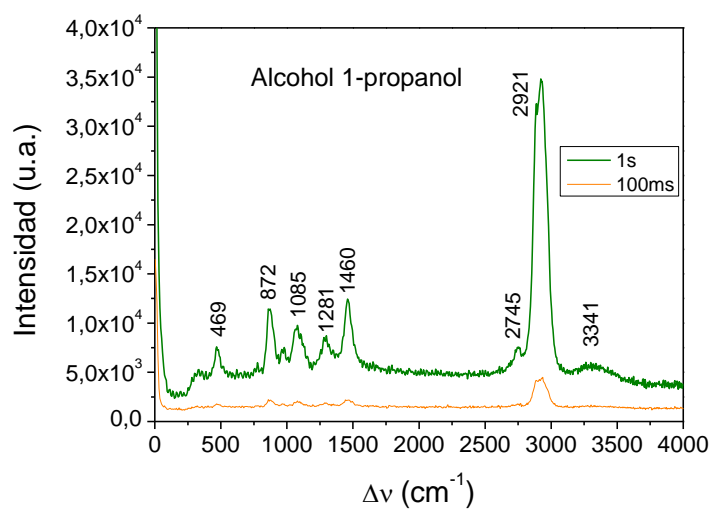

d)

Fig. 16. Espectros Raman de alcoholes evaluados con tiempos de integración de $100 \mathrm{~ms}$ y 1s. a) Alcohol etílico, b) Alcohol metílico, c) Alcohol 1-butanol, d) Alcohol 1-propanol.

\section{CONCLUSIONES}

Se ha desarrollado un prototipo de equipo de espectroscopía Raman para análisis cualitativo de muestras en polvo y muestras líquidas. El equipo tiene una resolución óptica de $1.4 \mathrm{~nm}\left(35 \mathrm{~cm}^{-1}\right.$ en unidades empleadas en espectroscopía molecular) y un rango espectral de 200 a $4000 \mathrm{~cm}^{-1}$. Estos parámetros dependen del sistema de detección instalado que en este trabajo es el espectrómetro ocean optics USB4000UV-VIS-ES, el cual tiene una rejilla de difracción de 600 líneas por mm, un rango espectral de 0-850 nm y apertura numérica de 0.22.

Se ha establecido un procedimiento de alineación para un arreglo Raman con geometría $180^{\circ}$ y con colección a muestras en dos ejes. Así mismo, se ha validado la alineación del arreglo mediante la detección de muestras con débil emisión Raman, éste fue el caso del óxido de zinc con bandas en $545 \mathrm{~nm}$ y $567 \mathrm{~nm}$ y del agua deshionozada con una banda centrada en $648 \mathrm{~nm}$. 


\section{Agradecimientos}

- El autor agradece la colaboración académica del Profesor Roberto Sato Berrú del Centro de Ciencias Aplicadas y Desarrollo Tecnológico de la Universidad Nacional Autónoma de México (CCADET-UNAM) y el apoyo económico de la Pontificia Universidad Católica del Perú (PUCP) para el desarrollo de una estancia de investigación en CCADET-UNAM.

- El autor agradece la beca otorgada por la Red Iberoamericana de Óptica que permitió mi participación en la IX Conferencia RIAO-OPTILAS 2016. 